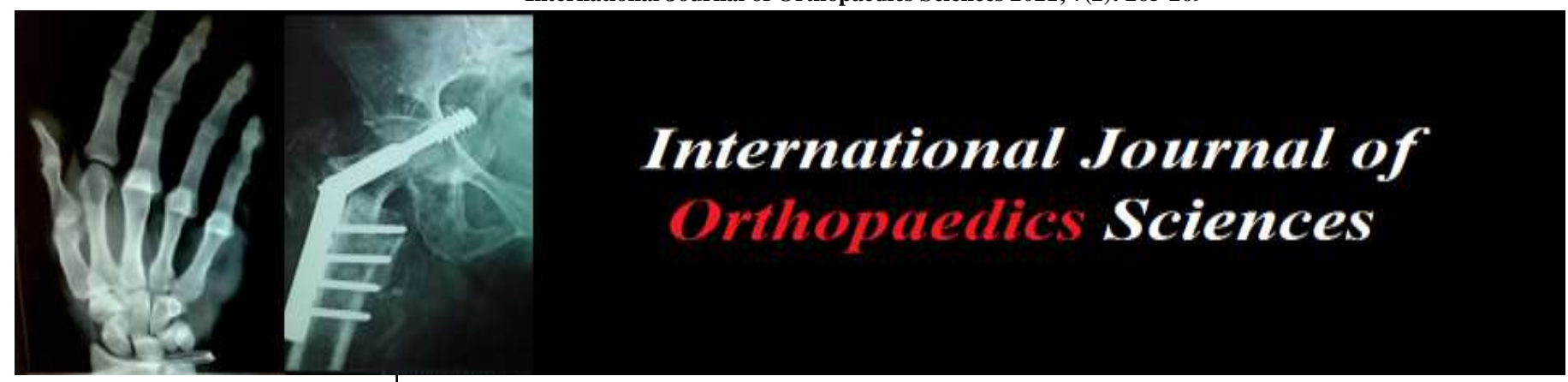

E-ISSN: 2395-1958

P-ISSN: 2706-6630

IJOS 2021; 7(2): 265-269

(C) 2021 IJOS

www.orthopaper.com

Received: 06-02-2021

Accepted: 16-03-2021

Dr. Aravind Subramanian MBBS, M.S (Ortho)

Kalinga Institute of Medical

Sciences, Kushabhadra Campus

KIIT Campus, 5, KIIT Road,

Patia Bhubaneswar, Odisha,

India

Dr. Amlan Dash

M.B.B.S, M.S Ortho, Kalinga Institute of Medical Sciences, Kushabhadra Campus,

KIIT Campus, 5, KIIT Road,

Patia, Bhubaneswar, Odisha, India

Dr. Konchada Srikant MBBS DNB Ortho. Kalinga Institute of Medical Sciences, Kushabhadra Campus

KIIT Campus, 5, KIIT Road, Patia, Bhubaneswar, Odisha, India

Dr. Berikai Venkat Aditiya Kalinga Institute of Medical Sciences, Kushabhadra Campus KIIT Campus, 5, KIIT Road, Patia, Bhubaneswar, Odisha, India

Dr. Eshwar Patel K Kalinga Institute of Medical Sciences, Kushabhadra Campus KIIT Campus, 5, KIIT Road, Patia Bhubaneswar, Odisha, India

\section{Study of various modalities of treatment of internal fixation of tibial condylar fracture-schatzkers type $v$ and type VI}

\author{
Dr. Aravind Subramanian, Dr. Amlan Dash, Dr. Konchada Srikant and \\ Dr. Berikai Venkat Aditiya
}

DOI: https://doi.org/10.22271/ortho.2021.v7.i2d.2641

\begin{abstract}
Fractures of the tibial plateau usually result from high energy trauma. Fractures of the tibial plateau change the knee kinematics, alter joint stability, and cause joint incongruity. Fractures range from simple lateral condyle fractures to severe comminuted metaphyseal fractures. Various surgical modalities like open reduction internal fixation with cannulated Screw fixation, Condylar plate with or without bone graft, AO/ASIF Buttress plate (T/L) with or without bone graft, Proximal tibial locking plate have been practiced. To study the functional outcomes of various modalities of treatment of internal fixation of tibial condylar fractures schatzkers type V and type V1. To find out the basis of selecting the mode of treatment of tibial condylar fractures to bring out the best functional results. 35 patients of age group 1870 yrs who presented with X-ray based Schatzker Type V and VI proximal tibia fractures were selected for the study. All the patients underwent Open Reduction and Internal Fixation with locking compression plating. The functional outcomes were assessed using knee society score. Complications were observed and treated accordingly with an average follow-up of 12 months. It was observed that the mean duration of the union was 14-16 weeks. Most of the patients (19) showed excellent results with a good range of motion of $122^{\circ}$. The combination of dual approaches with dual fixation and with fixed angle locking plates is a safe and effective way to have direct reduction and satisfactory fixation for complex tibial plateau fractures.
\end{abstract}

Keywords: Tibial plateau fracture, schatzkers, approaches, dual plating, knee society score

\section{Introduction}

Fractures of the tibial plateau usually result from high energy trauma. Motor vehicle accidents seem to be the predominant cause worldwide. The knee is the major weight-bearing joint of the body ${ }^{[1]}$ Fractures of the tibial plateau change the knee kinematics, alter joint stability and cause joint incongruity. Fractures range from simple injuries with predictably excellent outcomes after nonoperative treatment to complex fracture patterns that challenge even the most experienced surgeons ${ }^{[2]}$. Imaging studies need to be of good quality to demonstrate the location of the fracture, the fracture pattern, and the degree of displacement, and there is controversy on which type of imaging is optimal.

Less severe types can be treated operatively or non-operative. For more severe type fractures there is consensus regarding operative management. The various modalities of treatment aim at elevating the depressed articular surface, maintaining the elevation utilizing plates and screws, and if necessary filling the defect with bone graft to gain the maximum range of knee Motion ${ }^{[4]}$. Various surgical modalities like open reduction internal fixation with cannulated Screw fixation, Condylar plate with or without bone graft, AO/ASIF Buttress plate (T/L) with or without bone graft, Proximal tibial locking plate have been practiced. Biomechanical data showed four $3.5 \mathrm{~mm}$ screws were superior to two $6.5 \mathrm{~mm}$ screws in axial compression. Tibial plateau fractures are serious injuries that commonly result in functional impairment. The objective in treating displaced fractures is to restore the articular surface anatomy, repair of soft tissue injuries, and rigid internal fixation to enable early mobilization to obtain a painless and stable knee joint with a normal range of movements, New methods have changed the surgical management of both low-energy lateral plateau fractures and high-energy medial and
Corresponding Author: Dr. Amlan Dash M.B.B.S, M.S Ortho, Kalinga Institute of Medical Sciences, Kushabhadra Campus, KIIT Campus, 5, KIIT Road, Patia, Bhubaneswar, Odisha, India 
bicondylar fractures, but more data are necessary to determine if these new methods are improving patient outcomes or not.

\section{Materials and Methods}

Study Population: All the patients presenting to OPD or Emergency in KIMS, Bhubaneswar, with Proximal Tibia Fractures, admitted in Department of Orthopedics.

Inclusion Criteria: Patients above 18 years of age irrespective of gender, Displaced and undisplaced Schatzkers type V, VI and closed fractures,
Exclusion criteria: open compound fractures, Pathological fractures, bilateral fractures, Compartment syndrome, Adjacent joint injuries, Non-consenting patients on admission, all the patients underwent a standard radiologic protocol of $\mathrm{x}$ rays and $\mathrm{CT}$ scan and were classified as per the schatzkers classification. They underwent relevant pre and post procedure investigations following which they were subjected to surgical management. Post-operative complications if any were, they were looked after meticulously and functional outcome was assessed by Oxford knee score. The cases were followed up at interval of 1 month, 3 months, 6 months and 12 months, for a maximum of 2 years.
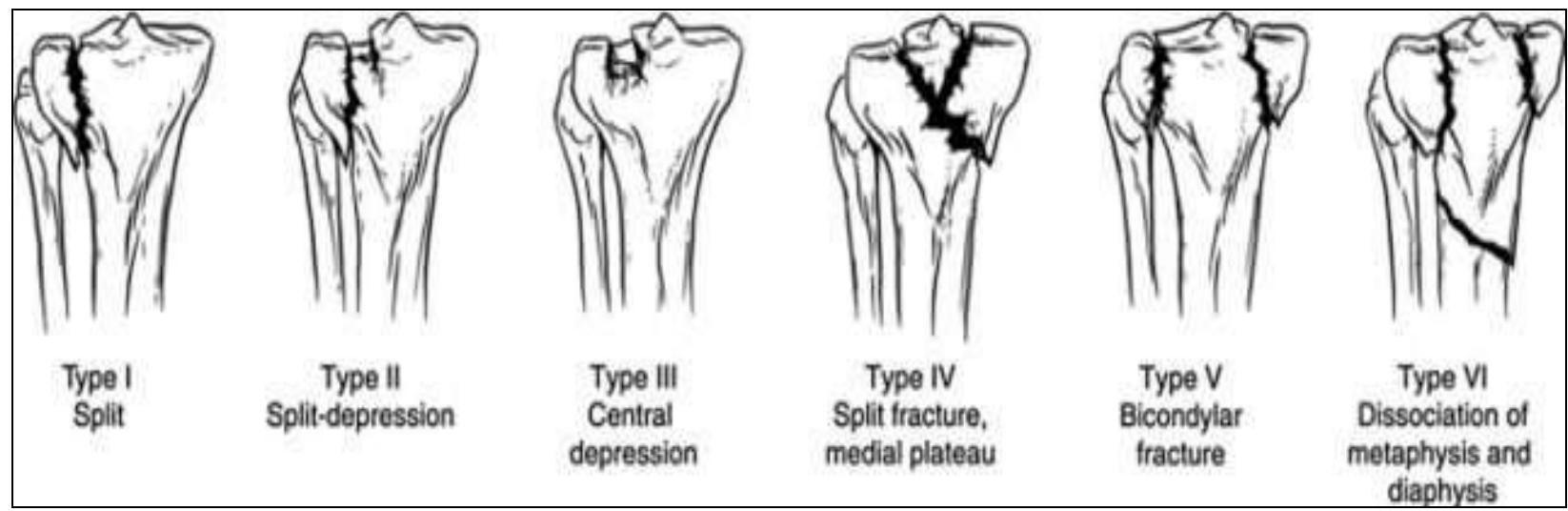

Fig 1: Schatzkers classification

\section{Surgical Procedure}

Patients were thoroughly investigated, affected knee, and the iliac crest was prepared. Surgery was done under spinal anesthesia. All patients were given Inj. Cefotaxime 1g IV preoperatively as routine prophylaxis. The patient was placed in the supine position, with a folded pillow under the knee to allow knee flexion. A femoral distractor was used whenever needed.

\section{Anterolateral Approach ${ }^{[4]}$}

Lateral plating is done using this approach. It is the workhorse approach for split depression fractures. The incision is based on Gerdy's tubercle and extended distally over the anterior compartment. An L-shaped incision over the origin of the anterior compartment muscles provides access to the anterolateral surface of the tibia. With the knee flexed in a varus and internally rotated position, the intraarticular damage was evaluated through a submeniscal arthrotomy. The fracture was mobilized with a chisel and reduced directly under vision; the articular surface depression was elevated and the fracture was reduced. The resulting subchondral or metaphyseal defect was then grafted with autograft. A large compression clamp was applied and the transverse diameter of the tibia was controlled under fluoroscopy. Finally, a lateral compression plate or buttress plate was used to stabilize the lateral compartment. This allows the mediolateral direction of the screw.

\section{Posteromedial Approach}

The posteromedial approach is used for medial plating. The incision was placed approximately $2 \mathrm{~cm}$ posterior to the posteromedial edge of the tibial shaft. The fascia overlying gastrocnemius was incised and the pes anserinus was retracted anteriorly. The intra-articular fracture-dislocation was visualized with a submeniscal arthrotomy. The impacted fragment was then mobilized in flexion and external rotation, which offers the best view on the posterior aspect of the tibia, and is reduced. In this position, a $3.5-\mathrm{mm}$ dynamic compression plate was contoured and fixed with screws in the distal fragment. The knee was then extended, and the posteromedial fragment is reduced with the $3.5-\mathrm{mm}$ plate acting as a dorsal buttress and was finally fixed with compression screws in a posteroanterior direction. The position of the screws was confirmed with an image intensifier before closing the wound.

Postoperative Instructions: Proper postoperative rehabilitation plays a major role in the recovery of range of movement and power the quadriceps mechanism and functions of the joint. If fracture fixation is stable, then physiotherapy can be started early and the most useful range of motion can be achieved, in the first few weeks of the postoperative period. Postoperative. Early post-op (1-3 weeks): Dressings of the wound. Physiotherapy: Encouraging knee extension, improving knee range of motion exercise. Quadricep strengthening, hamstring stretching, ankle pumping exercise.

Follow up Protocol -All patients were advised to review for regular follow up in the regular interval. A clinical, radiographical evaluation was done to assess the fracture healing at 1, 3, 6 \&12 months. 


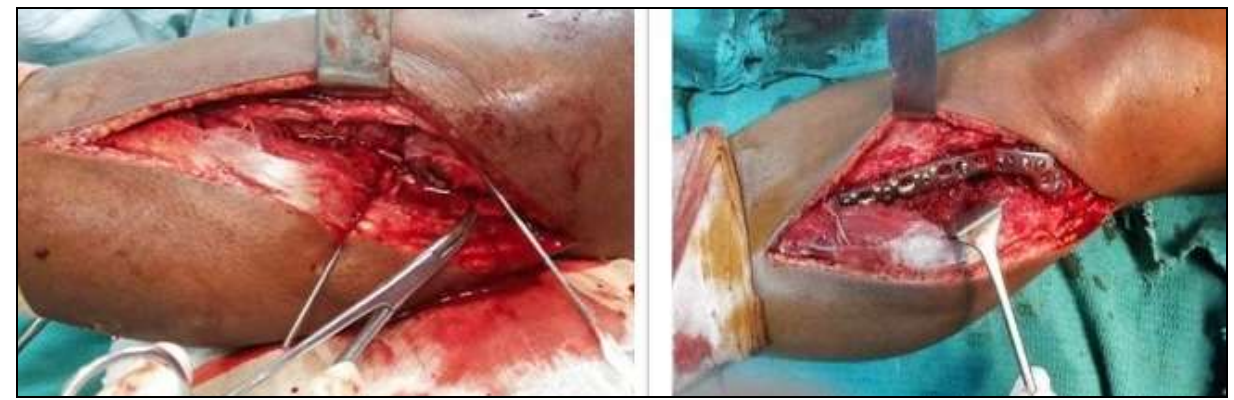

Fig 2: anterolateral approach (fracture exposed, reduction done and finally a lateral compression plate was used to stabilise

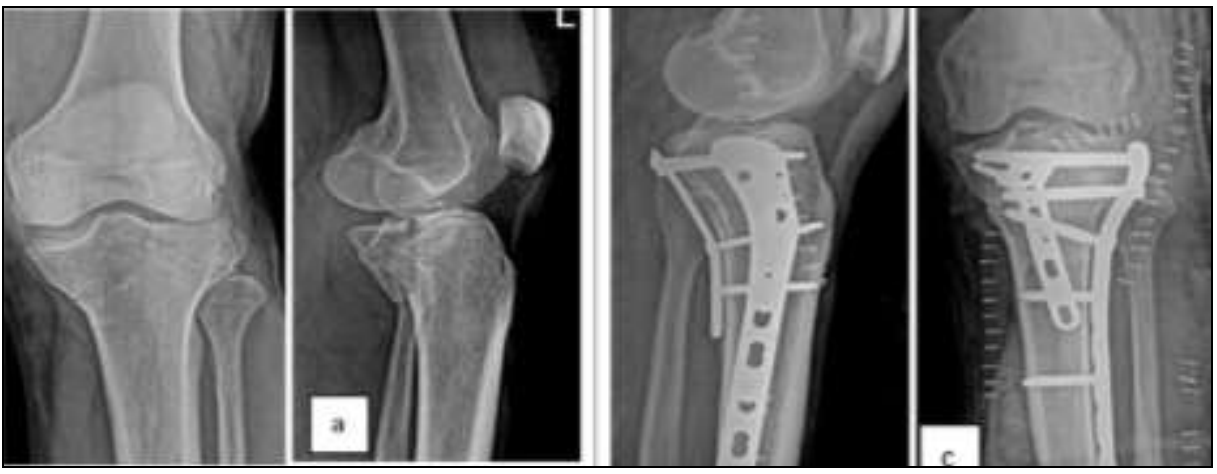

Fig 3: (a) showing pre op, (b) post op xray

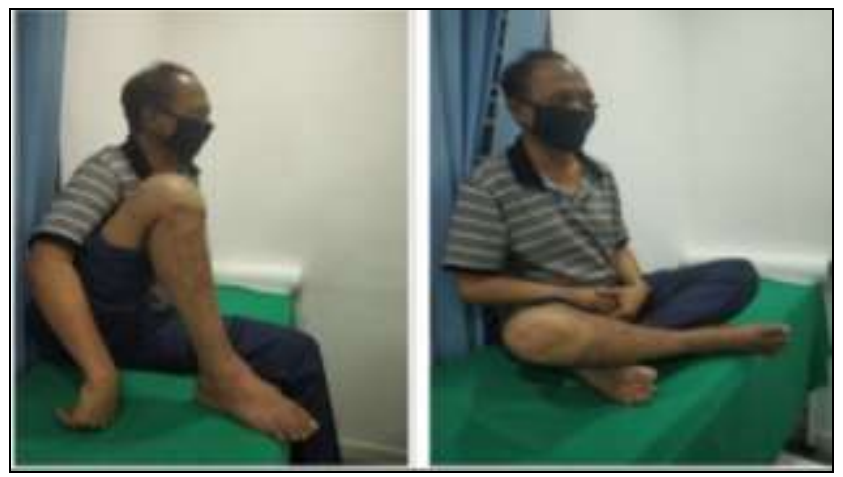

Fig 4: 9 months follow up

Table 1: showing mode of plating in type V and VI fractures

\begin{tabular}{|c|c|c|c|}
\hline S. No & Type of plating & type & No of pts \\
\hline \multirow{2}{*}{1} & \multirow{2}{*}{ Lateral plating only } & $\mathrm{V}$ & 13 \\
\hline & & VI & 1 \\
\hline \multirow{2}{*}{2} & \multirow{2}{*}{ Lateral plating and Medial plating (dual) } & $\mathrm{V}$ & 3 \\
\hline & & VI & 9 \\
\hline \multirow{2}{*}{3} & \multirow{2}{*}{$\begin{array}{l}\text { The lateral condyle and posteromedial } \\
\text { plating(dual) }\end{array}$} & $\mathrm{V}$ & 3 \\
\hline & & VI & 4 \\
\hline \multirow{2}{*}{4} & \multirow{2}{*}{ The lateral condyle and medial cc screws } & $\mathrm{V}$ & 2 \\
\hline & & VI & \\
\hline
\end{tabular}

Table 2: showing complication following post op

\begin{tabular}{|c|c|c|c|}
\hline S. No & Complication & Type & No of PTS \\
\hline \multirow{2}{*}{1} & \multirow{2}{*}{ Knee Stiffness } & $\mathrm{V}$ & \\
\hline & & VI & \\
\hline \multirow{2}{*}{2} & \multirow{2}{*}{ Varus Collapse } & $\mathrm{V}$ & \\
\hline & & VI & 3 \\
\hline \multirow{2}{*}{3} & \multirow{2}{*}{ Superficial Infection } & $\mathrm{V}$ & 2 \\
\hline & & VI & \\
\hline \multirow{2}{*}{4} & \multirow{2}{*}{ Nil } & $\mathrm{V}$ & - \\
\hline & & VI & - \\
\hline
\end{tabular}

Table 3: Showing knee society score of the post op patients

\begin{tabular}{|c|c|c|c|}
\hline S. No & KSS & No. PTS & \% \\
\hline 1 & Excellent $(85 \geq)$ & 19 & 54.2 \\
\hline 2 & Good $(70-84)$ & 11 & 31.4 \\
\hline 3 & Fair $(60-69)$ & 3 & 8.57 \\
\hline 4 & Poor $(<60)$ & 2 & 5.71 \\
\hline
\end{tabular}

\section{Results and Analysis}

In our study, 35 patients were assessed between September $1^{\text {st }}$ 2018 to august $31^{\text {st }} 2020$, they were classified based on schatzkers classification In our study, the tibial plateau fractures were most commonly due to RTA and between the mean age group 48.7 years .Out of 35 patients, $22(62.8 \%)$ were males and 13(37.2\%) were females. Type V were 22 $(62.8 \%)$ and type VI were $13(37.2 \%)$ There was a slight right-sided predominance $21(60 \%)$, compared to the left side`14 (40\%). All associated skeletal injuries were attended and given due care appropriately. 5 patients had Hypertension and 6 patients had Diabetes. Duration of hospitilisation of the patient was an average of 8.5 days. 14 patients (40\%) required bone grafting, and all were treated with autografting harvested from iliac crest. Single plating was done in 14 patients (40\%) dual plating was done in 19 patients $(54.2 \%)$ and single plating with CC screws was done in Two patient (5\%). All fractures united within expected time with an average time of 14.2 weeks. Average range of motion -122.9 degree .Not a single case of non-union was noted in our series.

The functional outcome of the post op patients scoring based on knee society score. The Average knee score was 86 . Out of 35 cases, we found varus collapse in 3 cases which continued throughout follow up. Two cases had a superficial infection at 6 months, out of which one resolved by antibiotics and the other re-quired debridement and then resolve. 


\section{Discussion}

Tibial plateau fractures especially type V and type VI pose a challenge to orthopaedic surgeons for being very complex, as they are associated with significant amount of comminution, compartment syndrome, severe soft tissue compromise, ligament injuries, and associated other bony injuries. Good surgical techniques and implants are essential for accurate articular reduction. Singleton et al. have shown that a postoperative step in articular joint surface of less than 2.5 $\mathrm{mm}$ indicate a good anatomical reduction which is pre requisite for a good clinical outcome. For such ideal fixation, intraoperative 3D Scan is needed, which was not possible in our study Due to inadequency of 3D Scan. So the aim towards treatment of these fracture are 'Good Anatomical reduction under visual and fluoroscopy, stable fixation and early mobilization for a painless, mobile joint 35 cases of Schatzker"s type V and type VI were studied and end results of this study are discussed below, taking into consideration of following parameters.

In our study, individuals in between 20-49 years are the ones who have maximum incidence of these high energy fractures, with average age group of 48.7years. Which correlates with study done by Vasanand et al. (2013) ${ }^{[5]}$ found $75 \%$ patient were in age group 30-50 years.

In our study $62.8 \%$ of the patients were males. This correlates well with the study done by $\mathrm{Wu}$ at al. (2015) ${ }^{[6]}$ found $75 \%$ male prevalence in their studies.

In our studies, type $\mathrm{V}$ fractures were $62.8 \%$ and type VI were $37.2 \%$. Wu et al (2015) ${ }^{[6]}$ found $55 \%$ prevalence of type $\mathrm{V}$ fractures.

Road traffic accident which are high velocity injuries was the commonest mode of injury $(65.2 \%)$ in my study. Lansinger ${ }^{[7]}$ reported $31 \%$ of the patients injured due to direct trauma, $33 \%$ due to fall from height and $45 \%$ were injured in road traffic accidents.

The union rate associated with RTA was lower compared around 16 weeks to other mode of injury fall around 13 weeks and fall from height around 14 weeks and this can be hypothesized to be due to increased extent of zone of injury. Neogi et al. ${ }^{[8]}$ Et showed similar out come with union rate of in RTA

In our study, there were $40 \%$ patients treated with use of bone graft (auto grafts) while $60 \%$ without use of the primary bone graft. Use of bone graft is necessary in such fractures as they involve significant amount of comminution and depression which need to be elevated to achieve articular congruous reduction. Use of bone grafting in form of autograft or allograft improve the outcome in treated fractures.

In our study, we have evaluated the patients functional outcomes using the knee society score which is a subjective score, and oxford knee score which is an objective score .where we found $55 \%$ excellent results, $32 \%$ good results, $8 \%$ fair and $5 \%$ poor results. These results are comparable to various other studies done. Chang-Wug Oh et al. ${ }^{[9]}$ reported excellent results in 21 patients out of 23 patients. In our Study, we observed that articular surface depression of 5-10 millimeters was well tolerated and did not affect the functional outcome on a short term follow up. The radiological values often do not co relate well with clinical outcomes. Which correletes with the study of Lucht and Pilgaard ${ }^{[10]}$ reviewed 109 fractures and found that most fractures with joint depression of 3-10 millimeters and even majority of fractures with more than 10 millimeters of joint depression had an acceptable functional result and varus collapse was found in 9 cases due to inadequate fixation, inadequate post op mobilsation and phsysiotherphy. The limitation of our study was a small study group and a relatively short period of follow up. A longer follow up would be necessary to evaluate the effect of articular surface depression as a factor in the development of osteoarthritis of the knee joint.

Barei DP, Nork SE, Mills WJ, Coles ${ }^{[11]}$ in their clinical study found most of the open reduction techniques were associated with high wound complication rates due to midline anterior approach or Mercedes-Benz incision. Reaching the posteromedial fragment through a single incision causes wide periosteal stripping and extensive muscle dissection and may hamper reduction as well. Dual incisions are better than single incision. In our study, dual incision with anterolateral and posteromedial approach was done for dual plating, the mean knee knee society score was found to be 86 . Average knee range of motion was 122.9 degree with no deep infection wound complication in any cases.

The advent of locking plates shifted the spectrum towards isolated lateral plating using locking compression plates and stabilizing medial fragment through screws passed via the locking plate to avoid varus collapse. Mohammad Ali Tahririan, Seyyed Hamid Mousavitadi, and Mohsen Derakhshan ${ }^{[12]}$ in their clinical study comparing the functional outcomes of tibial plateau fractures treated with nonlocking and locking plate fixation by knee society score, found a score of 80.2 for locking plate and 72.5 for non locking plate. Average range of knee flexion was found to be 122.3 degrees for locking plate and 115.7 degrees for non locking plate. In our study, locking plate was used for all the cases. Average knee society score was found to be 86 and average knee flexion was found to be 122.9 degrees. So, functional outcome in our study was marginally better than the locking plate group in that study and significantly better than the non locking group. This shows the superiority of the locking plate in view of stable fixation and early range of motion when compared to non locking plate.

The advantages of dual plate technique are visual reduction and maintenance of proximal tibial alignment, but soft tissue complications and damage to the periosteal blood supply are major concerns in this method of internal fixation. Some authors claimed a single lateral locking plate can decrease the risk of soft tissue damage and wound infection, but it cannot provide sufficient stability for multipart fractures.

In a biomechanical study by Mueller et al. ${ }^{[13]}$ stability in a tibial plateau fracture model was compared between the unilateral locking plate fixation and the dual locking plate fixation. The results were similar to those in the study of Yao et al. ${ }^{[14]}$. Who suggested that the shape of the medial condyle fracture plays a key role in single or double plate application. The posteromedial fracture fragment has been reported to have a frequency of $33 \%$ in bicondylar tibial plateau fractures. They reported there was no significant difference in the incidence of early postoperative malalignment and malreduction between dual plates and lateral locking plates used to treat bicondylar tibial plateau fractures in patients with a relatively intact medial condyle. Our study also had similar frequency with unilateral plating fixation for intact medial condyle and dual locking for comminuted or displaced medial and posteromedial fracture segment. Overall dual plate fixation followed commonly for complex tibial plateau fractures Different scoring systems have been recommended to evaluate functional results of tibial plateau fractures. We used KSS and OKS in this study. With regard to these systems, there were no difference between dual and single 
plating groups. In addition, in the literature, other series have reported good/excellent scores in $65 \%-89 \%$ of subjects ${ }^{[62]}$. Our results are comparable with those of other published studies with $86 \%$ satisfaction rates.

Ricci et al. and cole et al. reported about 9\% varus malalignment due to fixation and weight bearing and $4 \%$ Surgical Site infection. Varus complication is a rare and grave complication on long term outcome as it leads to early OA knee. Our study had same with three patients about $8.5 \%$ of the people, 2 due to early weight bearing and one due to inadequate fixation.

Lee et al. reported knee stiffness of about $9 \%$ due to Poor patient compliance. In our study had two cases i.e $4.3 \%$ of knee stiffness due to inadequate fixation of posteromedial fragment in one case and non compliant to physiotherapy in one case.

Weight-bearing was started only at 8-12 weeks which was similar to our study. Since our study group is small we were not able to statistically conclude which procedure is better. Choice of the procedure/implant should be based on the fracture pattern, bone quality, and intraoperative reduction

\section{Limitation}

The number of patients included in the study was 35 as per inclusion and exclusion criteria as these are injury due to increasing high velocity trauma but are associated with other injuries but isolated injury is commonly seen and duration of study was 2 year, with 1 year of recruitment and 1 year of minimum follow-up. Hence long term complications like osteoarthritis, deformity could not be assessed. These injuries are also sometimes associated with ligament and cartilage injury so preoperative MRI and intraoperative diagnostic arthroscopy could also be used for ligament and cartilage injury.

\section{Conclusion}

Schatzkers type $\mathrm{v}$ and VI Tibial plateau fractures are complex high energy injuries usually present with different and difficult configurations. With recent advancement in radiology like CT scan and MRI and evolution of arthroscopy, we need to incorporate the modern medicine for proper assessment of the injury and through understanding of the fracture pattern based on CT scan to plan surgical approach for adequate visual and fluoroscopic reduction.

The type of management with every fracture pattern changes as no fracture is the same, and should be managed on fracture to fracture basis. The surgical approach remains the important aspect and should be aided with through planning as Dual approach and dual fixation with respect to medial condyle and Fixation technique with fixed angle locking plates gives a better outcome and physiotherapy remain an intergral part for the post operative outcome.

\section{Reference}

1. Hohl M. Tibial condylar fractures. J. Bone Joint Surg., 1967;49A:1455-1467.

2. Apley AG. Fractures of the tibial plateau: The orthopedic clinics of North America 1978;10(1):61-74.

3. Koval KJ, Polatsch D, Kummer FJ, et al. Split fractures of the lateral tibial plateau: evaluation of three fixation methods. JOrthop Trauma 1996;10:304-308.

4. Gosling T, Muller M, Richter M et al. The less invasive stabilization system for bicondylar fractures of the proximal tibia. Presented at: Orthopaedic Trauma Association 18th Annual Meeting; Toronto, Canada.
5. Cambelles operative orthopaedics; Fractures of lower extremity: Tibial plateau 3:2094-2111.

6. Vasanad GH, Antin SM, Akkimaradi RC, Policepatil P, Naikawadi G. Surgical Management of Tibial Plateau Fractures - A Clinical Study. J Clin Diagn Res JCDR 2013;7(12):3128-30.

7. Wu D, Reng G, Shrivastava A, Yu Y, Zhang Y, Peng C. A useful surgical strategy for proximal tibial fractures (AO/OTA type 41-C) with diaphyseal involvement. Int J Clin Exp Med 2015;8(8):13455-63.

8. Lansinger O, Bergman B, KornerL. Tibial Condylar fractures, a twenty-year follow up. JBJS 1986;68:13-19.

9. Neogi DS, Trikha V, Mishra KK, Bandekar SM, Yadav CS. Comparative study of single lateral locked plating versus double plating in type $\mathrm{C}$ bicondylar tibial plateau fractures. Indian J Orthop 2015;49(2):193.

10. Oh CW, Oh JK, Kyung HS, Jeon IH, Park BC, Min WK, et al. Double plating of unstable proximal tibial fractures using minimally invasive percutaneous osteosynthesis technique. Acta Orthopaedica 2006;77(3):524-530. https://doi.org/10.1080/17453670610012548

11. Lucht U, Pilgaard S. Fractures of the tibial condyles. Acta Orthop Scand 1971:42:366-76.

12. Barei DP, Nork SE, Mills WJ, Coles CP, Henley MB, Benirschke SK. Functional outcomes of severe bicondylar tibial plateau fractures treated with dual incisions and medial and lateral plates. J Bone Joint Surg Am 2006;88:1713-21.

13. Clinical Study Comparison of Functional Outcomes of Tibial Plateau Fractures Treated with Nonlocking and Locking Plate Fixations: A Nonrandomized Clinical Trial. ISRN Orthopedics Volume March Article ID324573 Mohammad Ali Tahririan, Seyyed Hamid Mousavitadi, and Mohsen Derakhshan 2014.

14. Mueller KL, Karunakar MA, Frankenburg EP, Scott DS. Bicondylar tibial plateau fractures: a biomechanical study. Clin Orthop Relat Res 2003;(412):189-9.

15. A comparison of lateral fixation versus dual plating for simple bicondylar fractures Yunfeng Yao, Hao Lv, Junfeng Zan, Jisen Zhang 2015;22(3):2259. doi10.1016/j.knee.2015.02.002. 2015 Mar 5. 